\title{
Antiperiodic Solutions for a Kind of Nonlinear Duffing Equations with a Deviating Argument and Time-Varying Delay
}

\author{
Changjin $\mathrm{Xu}^{1}$ and Maoxin Liao ${ }^{2}$ \\ ${ }^{1}$ Guizhou Key Laboratory of Economics System Simulation, Guizhou University of Finance and Economics, Guiyang 550004, China \\ ${ }^{2}$ School of Mathematics and Physics, University of South China, Hengyang 421001, China \\ Correspondence should be addressed to Changjin Xu; xcj403@126.com
}

Received 13 May 2014; Revised 3 August 2014; Accepted 3 August 2014; Published 18 August 2014

Academic Editor: Shao-Ming Fei

Copyright (c) 2014 C. Xu and M. Liao. This is an open access article distributed under the Creative Commons Attribution License, which permits unrestricted use, distribution, and reproduction in any medium, provided the original work is properly cited.

\begin{abstract}
This paper deals with a kind of nonlinear Duffing equation with a deviating argument and time-varying delay. By using differential inequality techniques, some very verifiable criteria on the existence and exponential stability of antiperiodic solutions for the equation are obtained. Our results are new and complementary to previously known results. An example is given to illustrate the feasibility and effectiveness of our main results.
\end{abstract}

\section{Introduction}

In recent years, Duffing equations have attracted much attention due to its wide range of applications in many practical problems such as in physics, mechanics, and the engineering fields. Many results on various Duffing equations are available (see [1-12]). However, to the best of our knowledge, there are few results on the antiperiodic solutions of Duffing equations. Many authors argue that in many applied science fields the existence of antiperiodic solutions plays a key role in characterizing the behavior of nonlinear differential equations [13-36]. This motivates us to focus on the existence and stability of antiperiodic solutions for Duffing equations. In 2010, Peng and Wang [37] consider the existence of positive almost periodic solutions for the following nonlinear Duffing equation with a deviating argument:

$$
x^{\prime \prime}+c x^{\prime}-a x(t)+b x^{m}(t-\tau(t))=p(t),
$$

where $\tau(t)$ and $p(t)$ are almost periodic functions on $R, m>$ 1 , and $a, b$, and $c$ are constants. By applying some analysis technique, Peng and Wang [37] obtained the results on the existence of positive almost periodic solutions for system (1).

In this paper, we will consider the antiperiodic solutions of the following more general Duffing equation with a deviating argument and time-varying delay which takes the form

$$
x^{\prime \prime}+c(t) x^{\prime}-a(t) x(t)+b(t) x^{m}(t-\tau(t))=p(t),
$$

where $c(t), a(t), b(t)$, and $p(t)$ are continuous functions on $R$, $m>1$ is a constant, and $\tau(t) \geq 0$ is continuous functions on $R$. There exists a constant $\tau$ such that $\tau=\sup _{t \in R} \tau(t)$. By using differential inequality techniques, a series of new sufficient conditions for the existence, uniqueness, and exponential stability of antiperiodic solutions of system (2) are established. In addition, an example is presented to illustrate the effectiveness of our main results.

Let $a_{1}$ be a constant. Define

$$
y(t)=\frac{d x(t)}{d t}+a_{1} x(t) .
$$

Then system (2) can be transformed into the following equivalent system:

$$
\begin{aligned}
& \frac{d x(t)}{d t}=-a_{1} x(t)+y(t) \\
\frac{d y(t)}{d t}= & -\left(c(t)-a_{1}\right) y(t)+\left[a(t)-a_{1}\left(a_{1}-c(t)\right)\right] x(t) \\
& -b(t) x^{m}(t-\tau(t))+p(t)
\end{aligned}
$$


Let $\mathrm{BC}((-\infty, 0], R)$ denote the space of bounded continuous functions $\varphi:[-\infty, 0] \rightarrow R$ with the supremum norm $\|\cdot\|$. According to Burton [38], Hale [39], and Yoshizawa [40], we know that for $a(t), b(t), c(t)$, and $p(t)$ continuous, given a continuous initial function $\varphi \in \mathrm{BC}((-\infty, 0], R)$ and a vector $y_{0} \in R$, there exists a solution of (4) on an interval $[0, T)$ satisfying the initial condition and satisfying $(4)$ on $[0, T)$. If the solution remains bounded, then $T=+\infty$. We denote such a solution by $(x(t), y(t))=\left(x\left(t, \varphi, y_{0}\right), y\left(t, \varphi, y_{0}\right)\right)$. Let $y(s)=y(0)$ for all $s \in[-\tau, 0]$. It follows that $(x(t), y(t))$ can be defined on $[-\tau,+\infty]$.

Definition 1. Let $u(t): R \rightarrow R$ be continuous function in $t$. $u(t)$ is said to be $T$-antiperiodic on $R$ if

$$
u(t+T)=-u(t)
$$

for all $t \in R$.

Definition 2. Let $Z^{*}(t)=\left(x^{*}(t), y^{*}(t)\right)$ be an antiperiodic solution of (4) with initial value $\left(\varphi^{*}(t), y_{0}^{*}\right) \in \mathrm{BC}((-\infty, 0]$, $R) \times R \times R$. If there exist constants $\lambda>0$ and $M>1$ such that for every solution $Z(t)=(x(t), y(t))$ of (4) with an initial value $\varphi=\left(\varphi(t), y_{0}\right) \in \mathrm{BC}((-\infty, 0], R) \times R$,

$$
\begin{aligned}
& \max \left\{\left|x(t)-x^{*}(t)\right|,\left|y(t)-y^{*}(t)\right|\right\} \\
& \quad \leq M \max \left\{\left\|\varphi(t)-\varphi^{*}(t)\right\|,\left|y_{0}-y_{0}^{*}\right|\right\} e^{-\lambda t}
\end{aligned}
$$

for all $t>0$, where

$$
\left\|\varphi(t)-\varphi^{*}(t)\right\|=\sup _{t \in(-\infty, 0]}\left|\varphi(t)-\varphi^{*}(t)\right| .
$$

Then $Z^{*}(t)$ is said to be globally exponentially stable.

Throughout this paper, we make the following assumptions.

(H1) There exists a constant $T>0$ such that

$$
\begin{gathered}
a(t+T)=a(t), \quad b(t+T)=b(t), \\
c(t+T)=c(t), \quad \tau(t+T)=\tau(t), \\
p(t+T)=-p(t),
\end{gathered}
$$

for all $t, u \in R$.

(H2) There exists a constant $p^{+}$such that, for all $t>0$,

$$
p^{+}=\sup _{t \in R}|p(t)|
$$

(H3) There exists a constant $\lambda>0$ such that $\lambda-\left(a_{1}-1\right)<0$ and

$$
\begin{gathered}
\lambda-\inf _{t \in R}\left(c(t)-a_{1}\right)+\sup _{t \in R}\left|a(t)-a_{1}\left(a_{1}-c(t)\right)\right| \\
+b^{+}\left[(m-1) \delta^{m-1}+1\right] e^{\lambda \tau}<0 .
\end{gathered}
$$

The organization of this paper is as follows. In Section 2, we give some preliminary results. In Section 3, we derive the existence of $T$-antiperiodic solution, which is globally exponentially stable. An example is provided to illustrate the effectiveness of our main results in Section 4 .

\section{Preliminary Results}

In this section, we will first present two important lemmas which are used in what follows.

Lemma 3. Let (H1)-(H2) hold. Suppose that $(\tilde{x}(t), \tilde{y}(t))$ is a solution of (4) with initial conditions

$$
\tilde{x}(s)=\varphi(s), \quad \tilde{y}(s)=y_{0}, \quad \max \left\{|\tilde{x}(s)|,\left|y_{0}\right|\right\}<\delta,
$$

$$
s \in[-\tau, 0] \text {, }
$$

where $\delta$ satisfies

$$
\begin{gathered}
\inf _{t \in R}\left(c(t)-a_{1}\right) \delta-\sup _{t \in R}\left[a(t)-a_{1}\left(a_{1}-c(t)\right)\right] \delta \\
-b^{+} \delta^{m}-p^{+}>0 .
\end{gathered}
$$

Then,

$$
\max \{|\tilde{x}(t)|,|\tilde{y}(t)|\}<\delta
$$

for all $t \geq 0$.

Proof. By way of contradiction, we assume that (13) do not hold. Then one of the following two cases must occur.

Case 1. There exists $t_{1}>0$ such that

$$
\begin{gathered}
\max \left\{\left|\tilde{x}\left(t_{1}\right)\right|,\left|\tilde{y}\left(t_{1}\right)\right|\right\}=\left|\tilde{x}\left(t_{1}\right)\right|=\delta, \\
\max \{|\tilde{x}(t)|,|\tilde{y}(t)|\}<\delta,
\end{gathered}
$$

where $t \in\left[-\tau, t_{1}\right)$.

Case 2. There exists $t_{2}>0$ such that

$$
\begin{gathered}
\max \left\{\left|\tilde{x}\left(t_{2}\right)\right|,\left|\tilde{y}\left(t_{2}\right)\right|\right\}=\left|\tilde{y}\left(t_{2}\right)\right|=\delta, \\
\max \{|\tilde{x}(t)|,|\tilde{y}(t)|\}<\delta,
\end{gathered}
$$

where $t \in\left[-\tau, t_{2}\right)$.

If Case 1 holds true, we can calculate the upper left derivative of $|\tilde{x}(t)|$ as follows:

$$
0 \leq D^{+}\left(\left|\tilde{x}\left(t_{1}\right)\right|\right) \leq-a_{1}\left|x\left(t_{1}\right)\right|+\left|y\left(t_{1}\right)\right| \leq-\left(a_{1}-1\right) \delta<0,
$$

which is a contradiction. Then (13) holds. 
If Case 2 holds true, we can calculate the upper left derivative of $|\widetilde{y}(t)|$ as follows:

$$
\begin{aligned}
0 \leq & D^{+}\left(\left|\tilde{y}\left(t_{2}\right)\right|\right) \\
\leq & -\left(c\left(t_{2}\right)-a_{1}\right)\left|y\left(t_{2}\right)\right| \\
& +\mid\left[a\left(t_{2}\right)-a_{1}\left(a_{1}-c\left(t_{2}\right)\right)\right] x\left(t_{2}\right) \\
& \quad-b(t) x^{m}\left(t_{2}-\tau\left(t_{2}\right)\right)+p\left(t_{2}\right) \mid \\
\leq & -\inf _{t \in R}\left(c(t)-a_{1}\right)\left|y\left(t_{2}\right)\right| \\
& +\sup _{t \in R}\left[a(t)-a_{1}\left(a_{1}-c(t)\right)\right]\left|x\left(t_{2}\right)\right| \\
& +b^{+}\left|x^{m}\left(t_{2}-\tau\left(t_{2}\right)\right)\right|+p^{+} \\
\leq & -\left[\inf _{t \in R}\left(c(t)-a_{1}\right) \delta-\sup _{t \in R}\left[a(t)-a_{1}\left(a_{1}-c(t)\right)\right] \delta\right. \\
& \left.\quad-b^{+} \delta^{m}-p^{+}\right]<0,
\end{aligned}
$$

which is a contradiction. Then (13) holds.

Remark 4. It follows from the boundedness of this solution and the theory of functional differential equations in [36] that $(\widetilde{x}(t), \tilde{y}(t))$ can be defined on $[0,+\infty)$.

Lemma 5. Suppose that (H1)-(H3) hold. Let $Z^{*}(t)=\left(x^{*}(t)\right.$, $\left.y^{*}(t)\right)$ be the solution of (4) with initial values $\left(\varphi^{*}(s), y_{0}^{*}\right) \in$ $C([-\tau, 0], R) \times R$, and let $Z(t)=(x(t), y(t))$ be the solution of (4) with initial value $\left(\varphi(s), y_{0}\right) \in C([-\tau, 0], R) \times R$. Then there exists a constant $M>1$ such that

$$
\begin{aligned}
& \max \left\{\left|x(t)-x^{*}(t)\right|,\left|y(t)-y^{*}(t)\right|\right\} \\
& \quad \leq M \max \left\{\left\|\varphi(t)-\varphi^{*}(t)\right\|,\left|y_{0}-y_{0}^{*}\right|\right\} e^{-\lambda t}
\end{aligned}
$$

for all $t>0$.

Proof. Let $\bar{u}(t)=\left\{x(t)-x^{*}(t)\right\}, \bar{v}(t)=\left\{y(t)-y^{*}(t)\right\}$. Then,

$$
\begin{aligned}
& \frac{d \bar{u}(t)}{d t}=-a_{1} \bar{u}(t)+\bar{v}(t), \\
& \frac{d \bar{v}(t)}{d t}=-\left(c(t)-a_{1}\right) \bar{v}(t) \\
&+\left[a(t)-a_{1}\left(a_{1}-c(t)\right)\right] \bar{u}(t) \\
&-b(t)\left[x^{m}(t-\tau(t))-x^{* m}(t-\tau(t))\right] .
\end{aligned}
$$

In the sequel, we define the Lyapunov functional as follows:

$$
V_{1}(t)=|\bar{u}(t)| e^{\lambda t}, \quad V_{2}(t)=|\bar{v}(t)| e^{\lambda t} .
$$

Calculating the upper left derivative of $V_{i}(t)(i=1,2)$ along the solution $(\bar{u}(t), \bar{v}(t))$ of system (20) with the initial value $\left(\varphi(t)-\varphi^{*}(t), y_{0}-y_{0}^{*}\right)$, we have

$$
\begin{aligned}
& D^{+}\left(V_{1}(t)\right) \\
& \leq \lambda|\bar{u}(t)| e^{\lambda t}+D^{+}(|\bar{u}(t)|) e^{\lambda t} \\
& \leq \lambda|\bar{u}(t)| e^{\lambda t}+e^{\lambda t} \operatorname{sign}(\bar{u}(t))\left[-a_{1} \bar{u}(t)+\bar{v}(t)\right] \\
& \leq e^{\lambda t}\left[\left(\lambda-a_{1}\right)|\bar{u}(t)|+|\bar{v}(t)|\right], \\
& D^{+}\left(V_{2}(t)\right) \\
& \leq \lambda|\bar{v}(t)| e^{\lambda t}+D^{+}(|\bar{v}(t)|) e^{\lambda t} \\
& \leq \lambda|\bar{v}(t)| e^{\lambda t}+e^{\lambda t} \operatorname{sign}(\bar{v}(t)) \\
& \times\left\{-\left(c(t)-a_{1}\right) \bar{v}(t)\right. \\
& +\left[a(t)-a_{1}\left(a_{1}-c(t)\right)\right] \bar{u}(t)-b(t) \\
& \left.\times\left[x^{m}(t-\tau(t))-x^{* m}(t-\tau(t))\right]\right\} \\
& \leq e^{\lambda t}\left\{\left[\lambda-\left(c(t)-a_{1}\right)\right]|\bar{v}(t)|\right. \\
& +\sup _{t \in R}\left|a(t)-a_{1}\left(a_{1}-c(t)\right)\right||\bar{u}(t)| \\
& \left.+b^{+}\left[m \delta^{m-1}+1\right]|\bar{u}(t-\tau(t))|\right\} .
\end{aligned}
$$

Let $M>1$ be an arbitrary real number and set

$$
\varrho=\max \left\{\left\|\varphi-\varphi^{*}\right\|,\left|y_{0}-y_{0}^{*}\right|\right\}>0 .
$$

Then by (21), we have

$$
\begin{array}{r}
V_{1}(t)=|\bar{u}(t)| e^{\lambda t}<M \varrho, \quad V_{2}(t)=|\bar{v}(t)| e^{\lambda t}<M \varrho, \\
\forall t \in[-\tau, 0] .
\end{array}
$$

Thus we can claim that

$$
V_{1}(t)=|\bar{u}(t)| e^{\lambda t}<M \varrho, \quad V_{2}(t)=|\bar{v}(t)| e^{\lambda t}<M \varrho,
$$$$
\forall t>0 \text {. }
$$

Otherwise, one of the following cases must occur.

Case (a). There exists $T_{1}>0$ such that

$$
V_{1}\left(T_{1}\right)=M \varrho, \quad V_{i}(t)<M \varrho, \quad \forall t \in\left(-\tau, T_{1}\right), i=1,2 .
$$

Case (b). There exists $T_{2}>0$ such that

$$
V_{2}\left(T_{2}\right)=M \varrho, \quad V_{i}(t)<M \varrho, \quad \forall t \in\left(-\tau, T_{2}\right), i=1,2 .
$$

If Case (a) holds, then it follows from (21) and (26) that

$$
\begin{aligned}
0 & \leq D^{+}\left(V_{1}\left(T_{1}\right)\right) \\
& \leq\left(\lambda-a_{1}\right)\left|\bar{u}\left(T_{1}\right)\right| e^{\lambda T_{1}}+\left|\bar{v}\left(T_{1}\right)\right| e^{\lambda T_{1}} \\
& \leq\left[\lambda-\left(a_{1}-1\right)\right] M \varrho .
\end{aligned}
$$


Then,

$$
0 \leq \lambda-\left(a_{1}-1\right),
$$

which contradicts (H3). Then (25) holds.

If Case (b) holds, then it follows from (22) and (27) that

$$
\begin{aligned}
0 \leq & D^{+}\left(V_{2}\left(T_{2}\right)\right) \\
\leq & {\left[\lambda-\inf _{t \in R}\left(c(t)-a_{1}\right)\right]\left|\bar{v}\left(T_{2}\right)\right| e^{\lambda T_{2}} } \\
& +\sup _{t \in R}\left|a(t)-a_{1}\left(a_{1}-c(t)\right)\right|\left|\bar{u}\left(T_{2}\right)\right| e^{\lambda T_{2}} \\
& +b^{+}\left[m \delta^{m-1}+1\right]\left|\bar{u}\left(T_{2}-\tau\left(T_{2}\right)\right)\right| e^{\lambda\left(T_{2}-\tau\left(T_{2}\right)\right)} e^{\lambda \tau\left(T_{2}\right)} \\
\leq & {\left[\lambda-\inf _{t \in R}\left(c(t)-a_{1}\right)\right] M \varrho } \\
& +\sup _{t \in R}\left|a(t)-a_{1}\left(a_{1}-c(t)\right)\right| M \varrho \\
& +b^{+}\left[m \delta^{m-1}+1\right] M \varrho e^{\lambda \tau\left(T_{2}\right)} \\
\leq & \left\{\lambda-\inf _{t \in R}\left(c(t)-a_{1}\right)+\sup _{t \in R}\left|a(t)-a_{1}\left(a_{1}-c(t)\right)\right|\right. \\
& \left.+b^{+}\left[m \delta^{m-1}+1\right] e^{\lambda \tau}\right\} M \varrho .
\end{aligned}
$$

Then,

$$
\begin{aligned}
0 \leq & \lambda-\inf _{t \in R}\left(c(t)-a_{1}\right)+\sup _{t \in R}\left|a(t)-a_{1}\left(a_{1}-c(t)\right)\right| \\
& +b^{+}\left[m \delta^{m-1}+1\right] e^{\lambda \tau},
\end{aligned}
$$

which contradicts (H3). Then (25) holds. It follows that

$$
\begin{aligned}
& \max \left\{\left|x(t)-x^{*}(t)\right|,\left|y(t)-y^{*}(t)\right|\right\} \\
& \quad \leq M \max \left\{\left\|\varphi(t)-\varphi^{*}(t)\right\|,\left|y_{0}-y_{0}^{*}\right|\right\} e^{-\lambda t}
\end{aligned}
$$

for all $t>0$. This completes the proof of Lemma 5 .

Remark 6. If $Z^{*}(t)=\left(x^{*}(t), y^{*}(t)\right)$ is a $T$-antiperiodic solution of (4), it follows from Lemma 5 and Definition 2 that $Z^{*}(t)$ is globally exponentially stable.

\section{Main Results}

In this section, we present our main result that there exists the exponentially stable antiperiodic solution of (1).

Theorem 7. Assume that (H1)-(H3) are fulfilled. Then (4) with the initial condition (11) has exactly one T-antiperiodic solution $Z^{*}(t)=\left(x^{*}(t), y^{*}(t)\right)$. Moreover, this solution is globally exponentially stable.

Proof. Let $v(t)=\left(v_{1}(t), v_{2}(t)\right)=(x(t), y(t))$ be a solution of (4) with initial conditions (11). Thus according to Lemma 3 , the solution $v(t)$ is bounded and (13) holds. From (4), for any natural number $p$, we derive

$$
\begin{aligned}
& \left((-1)^{p+1} x(t+(p+1) T)\right)^{\prime} \\
& =(-1)^{p+1} x^{\prime}(t+(p+1) T) \\
& =(-1)^{p+1}\left[-a_{1} x(t+(p+1) T)+y(t+(p+1) T)\right] \\
& =-a_{1}(-1)^{p+1} x(t+(p+1) T) \\
& +(-1)^{p+1} y(t+(p+1) T), \\
& \left((-1)^{p+1} y(t+(p+1) T)\right)^{\prime} \\
& =(-1)^{p+1} y^{\prime}(t+(p+1) T) \\
& =(-1)^{p+1}\left\{-\left(c(t+(p+1) T)-a_{1}\right) y(t+(p+1) T)\right. \\
& +\left[a(t+(p+1) T)-a_{1}\left(a_{1}-c(t+(p+1) T)\right)\right] \\
& \times x(t+(p+1) T) \\
& -b(t+(p+1) T) x^{m} \\
& \times(t+(p+1) T-\tau(t+(p+1) T)) \\
& +p(t+(p+1) T)\} \\
& =-\left(c(t)-a_{1}\right)(-1)^{p+1} y(t+(p+1) T) \\
& +\left[a(t)-a_{1}\left(a_{1}-c(t)\right)\right](-1)^{p+1} x(t+(p+1) T) \\
& -b(t)(-1)^{p+1} x^{m}(t+(p+1) T-\tau(t))+p(t) \text {. }
\end{aligned}
$$

Thus $(-1)^{p+1} v(t+(p+1) T)$ are the solutions of (4) on $R$ for any natural number $p$. Then, from Lemma 5 , there exists a constant $M>1$ such that

$$
\begin{aligned}
& \left|(-1)^{p+1} v_{i}(t+(p+1) T)-(-1)^{k} v_{i}(t+p T)\right| \\
& \leq M e^{-\lambda(t+p T)} \sup _{-\tau \leq s \leq 0} \max _{1 \leq i \leq 2}\left|v_{i}(s+T)+v_{i}(s)\right| \\
& \leq 2 e^{-\lambda(t+p T)} M \delta, \quad \forall t+p T>0, \quad i=1,2 .
\end{aligned}
$$

Thus, for any natural number $q$, we have

$$
\begin{gathered}
(-1)^{q+1} v_{i}(t+(q+1) T) \\
=v_{i}(t)+\sum_{k=0}^{q}\left[(-1)^{k+1} v_{i}(t+(k+1) T)\right. \\
\left.-(-1)^{k} v_{i}(t+k T)\right] .
\end{gathered}
$$


Hence,

$$
\begin{gathered}
\left|(-1)^{q+1} v_{i}(t+(q+1) T)\right| \\
\leq\left|v_{i}(t)\right|+\sum_{k=0}^{q} \mid(-1)^{k+1} v_{i}(t+(k+1) T) \\
-(-1)^{k} v_{i}(t+k T) \mid,
\end{gathered}
$$

where $i=1,2$. By (35), we can choose a sufficiently large constant $N>0$ and a positive constant $\gamma$ such that

$$
\begin{array}{r}
\left|(-1)^{p+1} v_{i}(t+(p+1) T)-(-1)^{k} v_{i}(t+p T)\right| \leq \gamma\left(e^{-\lambda T}\right)^{k}, \\
\forall k>N, \quad i=1,2,
\end{array}
$$

on any compact set of $R$. It follows from (36) and (37) that $\left\{(-1)^{q} v(t+q T)\right\}$ uniformly converges to a continuous function $Z^{*}(t)=\left(x^{*}(t), y^{*}(t)\right)$ on any compact set of $R$.

Now we show that $Z^{*}(t)$ is $T$-antiperiodic solution of (4). Firstly, $Z^{*}(t)$ is $T$-antiperiodic, since

$$
\begin{aligned}
Z^{*}(t+T) & =\lim _{q \rightarrow \infty}(-1)^{q} v(t+T+q T) \\
& =-\lim _{(q+1) \rightarrow \infty}(-1)^{q+1} v(t+(q+1) T)=-Z^{*}(t) .
\end{aligned}
$$

In the sequel, we prove that $Z^{*}(t)$ is a solution of (4). Noting that the right-hand side of (4) is continuous, (33) shows that $\left\{\left((-1)^{q+1} v(t+(q+1) T)\right)^{\prime}\right\}$ uniformly converges to a continuous function on any compact subset of $R$. Thus, letting $q \rightarrow \infty$ on both sides of (33), we can easily obtain

$$
\begin{gathered}
\frac{d x^{*}(t)}{d t}=-a_{1} x^{*}(t)+y^{*}(t) \\
\frac{d y^{*}(t)}{d t}=-\left(c(t)-a_{1}\right) y^{*}(t) \\
+\left[a(t)-a_{1}\left(a_{1}-c(t)\right)\right] x^{*}(t) \\
-b(t) x^{* m}(t-\tau(t))+p(t) .
\end{gathered}
$$

Therefore, $Z^{*}(t)$ is a solution of (4). Applying Lemma 5, we can easily check that $Z^{*}(t)$ is globally exponentially stable. The proof of Theorem 7 is completed.

\section{An Example}

In this section, we give an example to illustrate our main results obtained in previous sections.

Example 1. The following two-order Duffing equation with two deviating arguments,

$$
\begin{aligned}
x^{\prime \prime}+ & (10+|\sin t|) x^{\prime}-(0.5+|\cos t|) x(t) \\
& +0.2 \sin t x^{2}(t-0.01|\sin t|)=0.2 \sin t,
\end{aligned}
$$

has exactly one $\pi$-antiperiodic solution.

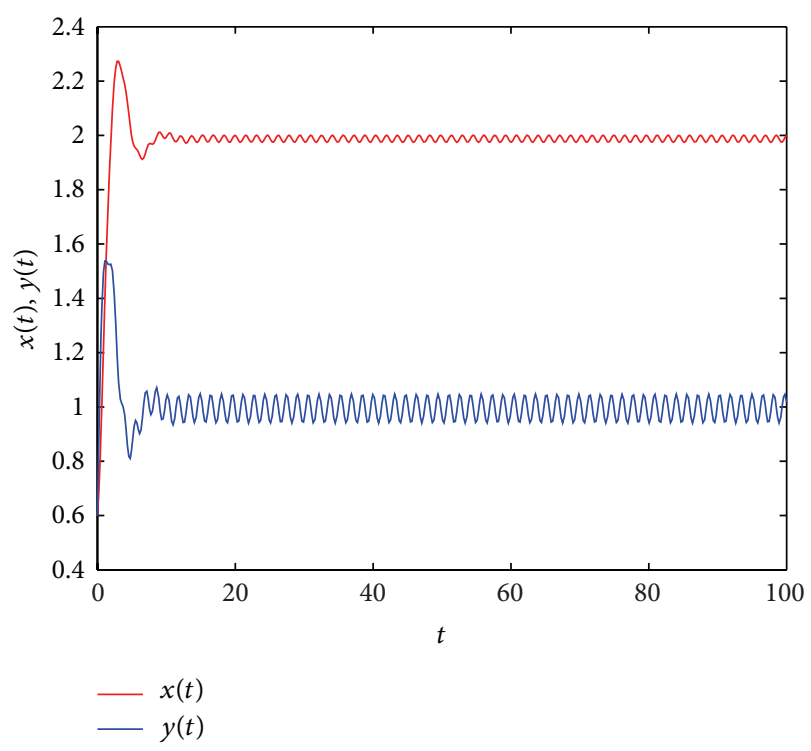

FIgURE 1: Time response of state variables $x$ and $y$.

Proof. Let

$$
y(t)=\frac{d x(t)}{d t}+0.5 x(t) .
$$

Then system (40) can be transformed into the following equivalent system:

$$
\begin{aligned}
& \frac{d x(t)}{d t}=-0.5 x(t)+y(t), \\
& \begin{aligned}
\frac{d y(t)}{d t} \\
=-(10+|\sin t|-0.5) y(t) \\
\quad+[0.5+|\cos t|-0.5(0.5-(10+|\sin t|))] x(t) \\
\quad-0.2 \sin t x^{2}(t-0.01|\sin t|)+0.2 \sin t .
\end{aligned}
\end{aligned}
$$

Corresponding to system (3) and (4), we have

$$
\begin{gathered}
a_{1}=0.5, \quad a(t)=0.5+|\cos t|, \quad b(t)=0.2 \sin t, \\
c(t)=10+|\sin t|, \quad m=2, \quad p(t)=0.2 \sin t, \\
\tau(t)=0.01|\sin t| .
\end{gathered}
$$

Let $\delta=0.5, \lambda=0.02$. Then $\tau=0.01, p^{+}=0.2$. It is easy to check that all the conditions in Theorem 7 are fulfilled. Hence we can conclude that system (42) has exactly one $\pi$ antiperiodic solution. Moreover, this $\pi$-periodic solution is globally exponentially stable. Thus system (40) has exactly one $\pi$-antiperiodic solution, and all solutions of system (40) exponentially converge to this $\pi$-antiperiodic solution. This result is illustrated in Figure 1. 


\section{Conflict of Interests}

The authors declare that there is no conflict of interests regarding the publication of this paper.

\section{Acknowledgments}

This work is supported by National Natural Science Foundation of China (no. 11261010, no. 11201138), Soft Science and Technology Program of Guizhou Province (no. 2011LKC2030), Natural Science and Technology Foundation of Guizhou Province (J[2012]2100), Governor Foundation of Guizhou Province ([2012]53), Natural Science and Technology Foundation of Guizhou Province (2014), Scientific Research Fund of Hunan Provincial Education Department (no. 12B034), and Natural Science Innovation Team Project of Guizhou Province ([2013]14).

\section{References}

[1] Z. B. Cheng and J. L. Ren, "Harmonic and subharmonic solutions for superlinear damped Duffing equation," Nonlinear Analysis: Real World Applications, vol. 14, no. 2, pp. 1155-1170, 2013.

[2] M. U. Akhmet and M. O. Fen, "Chaotic period-doubling and OGY control for the forced Duffing equation," Communications in Nonlinear Science and Numerical Simulation, vol. 17, no. 4, pp. 1929-1946, 2012.

[3] V. Marinca and N. HeriŞanu, "Explicit and exact solutions to cubic DUFfing and double-well DUFfing equations," Mathematical and Computer Modelling, vol. 53, no. 5-6, pp. 604-609, 2011.

[4] Y. Wang, "Novel existence and uniqueness criteria for periodic solutions of a Duffing type p-Laplacian equation," Applied Mathematics Letters, vol. 23, no. 4, pp. 436-439, 2010.

[5] B. Du, C. Bai, and X. Zhao, "Problems of periodic solutions for a type of Duffing equation with state-dependent delay," Journal of Computational and Applied Mathematics, vol. 233, no. 11, pp. 2807-2813, 2010.

[6] J. Llibre and A. Rodrigues, "A note on the periodic orbits of a kind of Duffing equations," Applied Mathematics and Computation, vol. 219, no. 15, pp. 8358-8365, 2013.

[7] Y.-K. Li and L. Yang, "Anti-periodic solutions for CohenGrossberg neural networks with bounded and unbounded delays," Communications in Nonlinear Science and Numerical Simulation, vol. 14, no. 7, pp. 3134-3140, 2009.

[8] B. Ahmad and B. S. Alghamdi, "Approximation of solutions of the nonlinear Duffing equation involving both integral and non-integral forcing terms with separated boundary conditions," Computer Physics Communications, vol. 179, no. 6, pp. 409-416, 2008.

[9] Q. Y. Zhou and B. W. Liu, "New results on almost periodic solutions for a class of nonlinear Duffing equations with a deviating argument," Applied Mathematics Letters, vol. 22, no. 1, pp. 6-11, 2009.

[10] L.-Q. Peng, "Existence and uniqueness of periodic solutions for a kind of Duffing equation with two deviating arguments," Mathematical and Computer Modelling, vol. 45, no. 3-4, pp. 378386, 2007.

[11] Q. Zhou and B. Liu, "New results on almost periodic solutions for a class of nonlinear Duffing equations with a deviating argument," Applied Mathematics Letters, vol. 22, no. 1, pp. 6-11, 2009.

[12] Y. Xu, "Positive almost periodic solutions for a class of nonlinear Duffing equations with a deviating argument," Electronic Journal of Qualitative Theory of Differential Equations, no. 80, pp. $1-9,2012$.

[13] J. Y. Shao, "Anti-periodic solutions for shunting inhibitory cellular neural networks with time-varying delays," Physics Letters A: General, Atomic and Solid State Physics, vol. 372, no. 30, pp. 5011-5016, 2008.

[14] Q. Fan, W. Wang, and X. Yi, "Anti-periodic solutions for a class of nonlinear $n$ th-order differential equations with delays," Journal of Computational and Applied Mathematics, vol. 230, no. 2, pp. 762-769, 2009.

[15] T. Zhang, Y. Li, and E. Xu, "Existence and stability of antiperiodic solution for a class of generalized neural networks with impulses and arbitrary delays on time scales," Journal of Inequalities and Applications, vol. 2010, Article ID 132790, 19 pages, 2010.

[16] A. R. Aftabizadeh, S. Aizicovici, and N. H. Pavel, "On a class of second-order anti-periodic boundary value problems," Journal of Mathematical Analysis and Applications, vol. 171, no. 2, pp. 301-320, 1992.

[17] S. Aizicovici, M. McKibben, and S. Reich, "Anti-periodic solutions to nonmonotone evolution equations with discontinuous nonlinearities," Nonlinear Analysis, Theory, Methods and Applications, vol. 43, no. 2, pp. 233-251, 2001.

[18] S. Gong, "Anti-periodic solutions for a class of CohenGrossberg neural networks," Computers and Mathematics with Applications, vol. 58, no. 2, pp. 341-347, 2009.

[19] B. Liu, "An anti-periodic LaSalle oscillation theorem for a class of functional differential equations," Journal of Computational and Applied Mathematics, vol. 223, no. 2, pp. 1081-1086, 2009.

[20] C. Ou, "Anti-periodic solutions for high-order Hopfield neural networks," Computers and Mathematics with Applications, vol. 56, no. 7, pp. 1838-1844, 2008.

[21] G. Peng and L. Huang, "Anti-periodic solutions for shunting inhibitory cellular neural networks with continuously distributed delays," Nonlinear Analysis: Real World Applications, vol. 10, no. 4, pp. 2434-2440, 2009.

[22] Z. Huang, L. Peng, and M. Xu, "Anti-periodic solutions for high-order cellular neural networks with time-varying delays," Electronic Journal of Differential Equations, vol. 2010, no. 5, pp. $1-9,2010$.

[23] A. P. Zhang, "Existence and exponential stability of antiperiodic solutions for HCNNs with timevarying leakage delays," Advances in Difference Equations, vol. 2013, article 162, 2013.

[24] Y. Li, L. Yang, and W. Wu, "Anti-periodic solutions for a class of Cohen-Grossberg neutral networks with time-varying delays on time scales," International Journal of Systems Science, vol. 42, no. 7, pp. 1127-1132, 2011.

[25] L. Pan and J. Cao, "Anti-periodic solution for delayed cellular neural networks with impulsive effects," Nonlinear Analysis: Real World Applications, vol. 12, no. 6, pp. 3014-3027, 2011.

[26] Y. Li and J. Shu, "Anti-periodic solutions to impulsive shunting inhibitory cellular neural networks with distributed delays on time scales," Communications in Nonlinear Science and Numerical Simulation, vol. 16, no. 8, pp. 3326-3336, 2011.

[27] Q. Fan, W. Wang, X. Yi, and L. Huang, "Anti-periodic solutions for a class of third-order nonlinear differential equations with a deviating argument," Electronic Journal of Qualitative Theory of Differential Equations, vol. 8, no. 12, pp. 1-12, 2011. 
[28] W. Wang and J. Shen, "Existence of solutions for anti-periodic boundary value problems," Nonlinear Analysis: Theory, Methods \& Applications, vol. 70, no. 2, pp. 598-605, 2009.

[29] Y. Chen, J. J. Nieto, and D. O’Regan, “Anti-periodic solutions for fully nonlinear first-order differential equations," Mathematical and Computer Modelling, vol. 46, no. 9-10, pp. 1183-1190, 2007.

[30] L. Peng and W. Wang, "Anti-periodic solutions for shunting inhibitory cellular neural networks with time-varying delays in leakage terms," Neurocomputing, vol. 111, pp. 27-33, 2013.

[31] J. Y. Park and T. G. Ha, "Existence of anti-periodic solutions for quasilinear parabolic hemivariational inequalities," Nonlinear Analysis: Theory, Methods \& Applications, vol. 71, no. 7-8, pp. 3203-3217, 2009.

[32] Y. Yu, J. Shao, and G. Yue, "Existence and uniqueness of antiperiodic solutions for a kind of Rayleigh equation with two deviating arguments," Nonlinear Analysis, Theory, Methods and Applications, vol. 71, no. 10, pp. 4689-4695, 2009.

[33] X. Lv, P. Yan, and D. Liu, "Anti-periodic solutions for a class of nonlinear second-order Rayleigh equations with delays," Communications in Nonlinear Science and Numerical Simulation, vol. 15, no. 11, pp. 3593-3598, 2010.

[34] Y. Li and L. Huang, "Anti-periodic solutions for a class of Liénard-type systems with continuously distributed delays," Nonlinear Analysis: Real World Applications, vol. 10, no. 4, pp. 2127-2132, 2009.

[35] B. W. Liu, "Anti-periodic solutions for forced Rayleigh-type equations," Nonlinear Analysis: Real World Applications, vol. 10, no. 5, pp. 2850-2856, 2009.

[36] P. Shi and L. Dong, "Existence and exponential stability of antiperiodic solutions of Hopfield neural networks with impulses," Applied Mathematics and Computation, vol. 216, no. 2, pp. 623630, 2010.

[37] L. Peng and W. Wang, "Positive almost periodic solutions for a class of nonlinear Duffing equations with a deviating argument," Electronic Journal of Qualitative Theory of Differential Equations, vol. 6, pp. 1-12, 2010.

[38] T. A. Burton, Stability and Periodic sSolutions of Ordinary and Functional Differential Equations, Academic Press, Orlando, Fla, USA, 1985.

[39] J. K. Hale, Theory of Functional Differential Equations, Springer, New York, NY, USA, 1977.

[40] T. Yoshizawa, "Asymptotic behaviors of solutions of differential equations," in Differential Equations: Qualitative Theory (Szeged, 1984), vol. 47 of Colloquia Mathematica Societatis János Bolyai, pp. 1141-1164, North-Holland, Amsterdam, The Netherlands, 1987. 


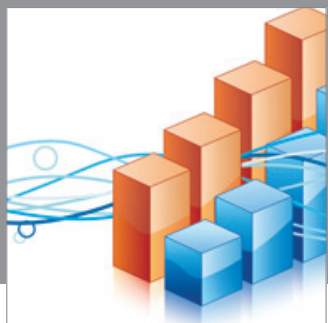

Advances in

Operations Research

mansans

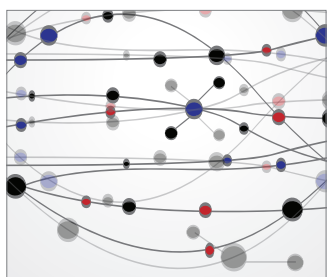

The Scientific World Journal
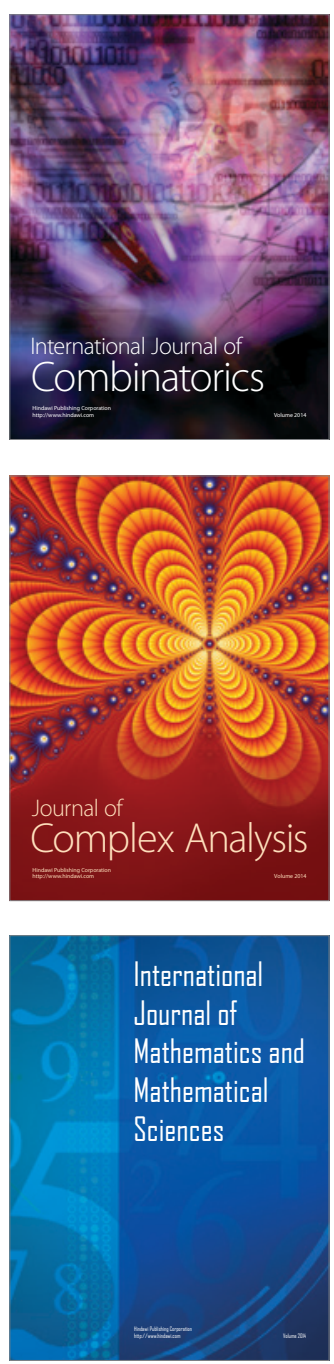
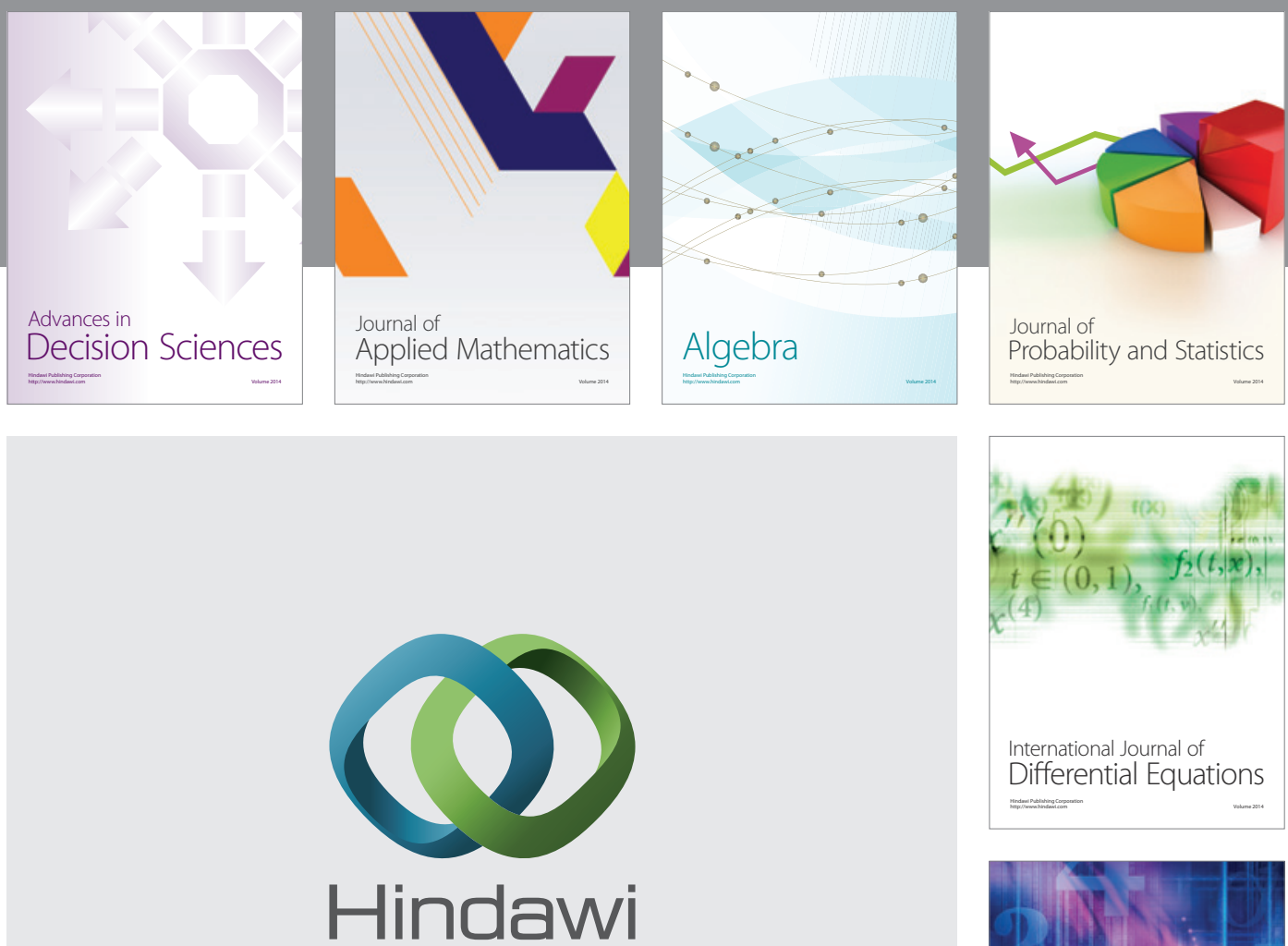

Submit your manuscripts at http://www.hindawi.com
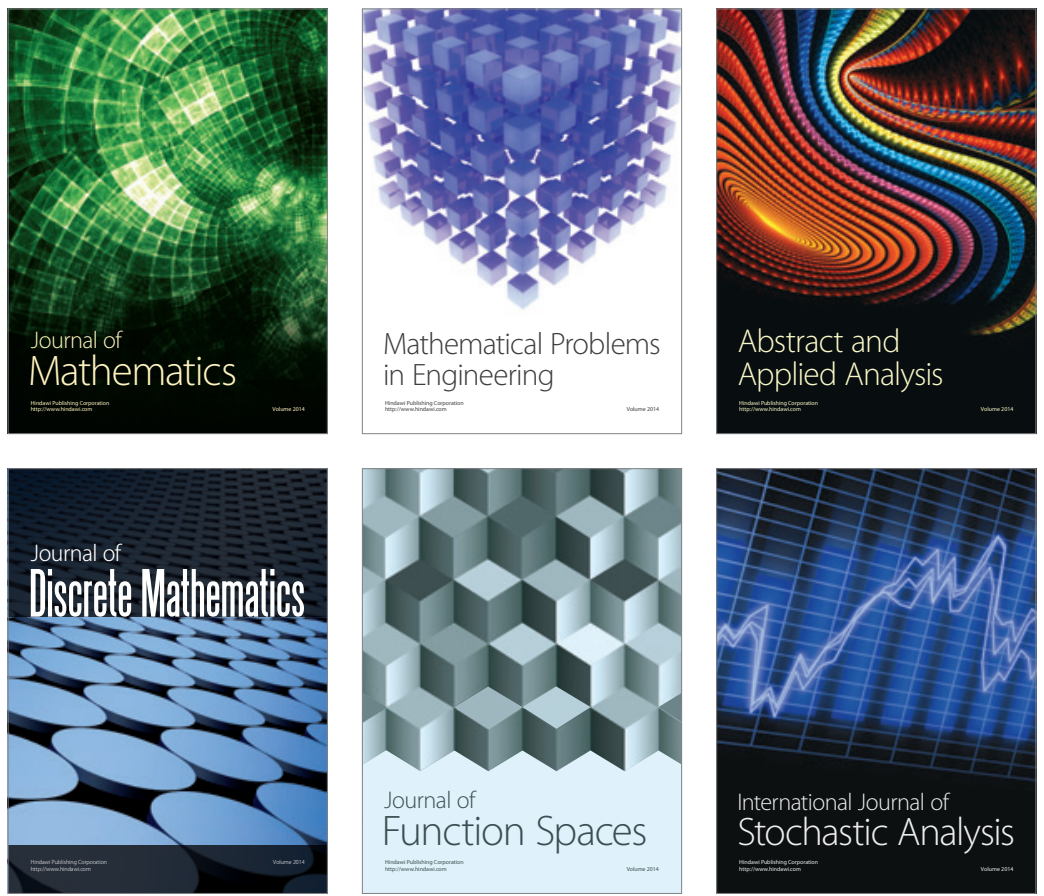

Journal of

Function Spaces

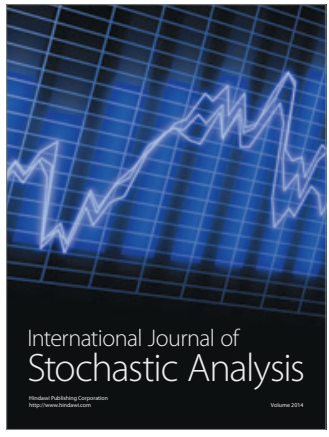

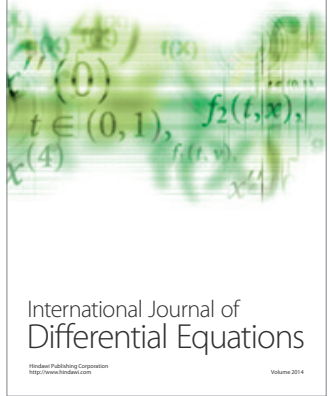
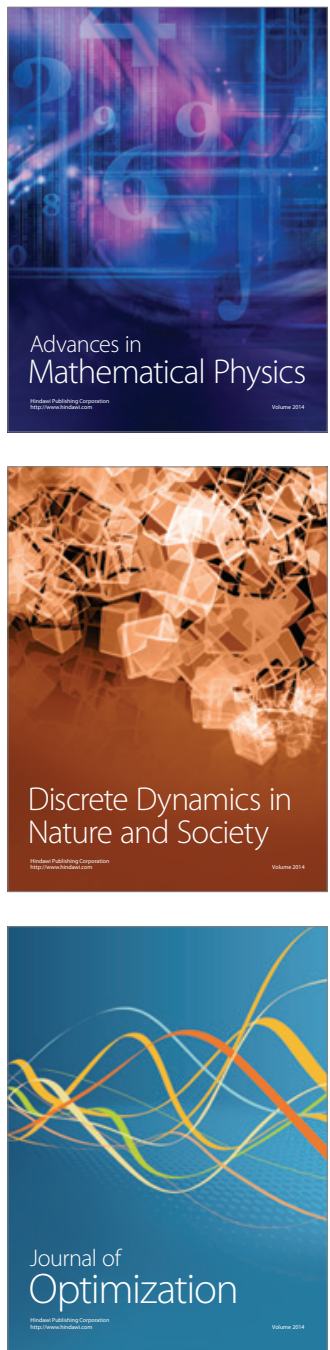